\title{
Increased reward in ankle robotics training enhances motor control and cortical efficiency in stroke
}

\author{
Ronald N. Goodman, PhD; ${ }^{*}$ Jeremy C. Rietschel, PhD; $^{1}$ Anindo Roy, PhD; ${ }^{1-2}$ Brian C. Jung, BS; ${ }^{1,3}$ Jason $^{1}$ \\ Diaz, MS; ${ }^{1}$ Richard F. Macko, MD; ${ }^{1-2}$ Larry W. Forrester, PhD $^{1,3}$ \\ ${ }^{1}$ Maryland Exercise and Robotics Center of Excellence, Baltimore Department of Veterans Affairs Medical Center, \\ Baltimore, MD; Departments of ${ }^{2}$ Neurology and ${ }^{3}$ Physical Therapy and Rehabilitation Science, University of Mary- \\ land School of Medicine, Baltimore, $M D$
}

\begin{abstract}
Robotics is rapidly emerging as a viable approach to enhance motor recovery after disabling stroke. Current principles of cognitive motor learning recognize a positive relationship between reward and motor learning. Yet no prior studies have established explicitly whether reward improves the rate or efficacy of robotics-assisted rehabilitation or produces neurophysiologic adaptations associated with motor learning. We conducted a 3 wk, 9-session clinical pilot with 10 people with chronic hemiparetic stroke, randomly assigned to train with an impedance-controlled ankle robot (anklebot) under either high reward (HR) or low reward conditions. The $1 \mathrm{~h}$ training sessions entailed playing a seated video game by moving the paretic ankle to hit moving onscreen targets with the anklebot only providing assistance as needed. Assessments included paretic ankle motor control, learning curves, electroencephalograpy (EEG) coherence and spectral power during unassisted trials, and gait function. While both groups exhibited changes in EEG, the HR group had faster learning curves $(p=0.05)$, smoother movements $(p</=0.05)$, reduced contralesional-frontoparietal coherence $(p</=0.05)$, and reduced left-temporal spectral power $(p</=0.05)$. Gait analyses revealed an increase in nonparetic step length $(p=0.05)$ in the HR group only. These results suggest that combining explicit rewards with novel anklebot training may accelerate motor learning for restoring mobility.
\end{abstract}

Clinical Trial Registration: ClinicalTrials.gov; NCT01072032; "Cortical and biomechanical dynamics of ankle robotics training in stroke"; http://www.clinicaltrials.gov/ct2/show/NCT01072032
Key words: anklebot, ankle robotics, chronic hemiparetic stroke, cognitive motor learning, EEG, EEG coherence, EEG spectral power, gait, plasticity, reward.

\section{INTRODUCTION}

Stroke is a major contributor to chronic disability worldwide. The sequelae often include loss of mobility, increased risk of falling, and cognitive impairment [1-3]. In particular for the majority of people with hemiparetic stroke, gait is a persistent problem [1-2,4-8].

Mounting evidence suggests that lower-limb (LL) motor-learning based interventions can improve movement function even years after a debilitating stroke [8-17]. This notion is supported by reports that treadmill-based

\footnotetext{
Abbreviations: $\mathrm{ANOVA}=$ analysis of variance, $\mathrm{CNS}=$ central nervous system, $\mathrm{DF}=$ dorsiflexion, $\mathrm{DOF}=$ degree of freedom, $\mathrm{EEG}=$ electroencephalograpy, $\mathrm{HR}=$ high reward, $\mathrm{ICA}=$ independent component analysis, $\mathrm{LL}=$ lower limb, $\mathrm{LR}=$ low reward, $\mathrm{PF}=$ plantar flexion, $\mathrm{VA}=$ Department of Veterans Affairs.

*Address all correspondence to Ronald N. Goodman, PhD; Baltimore VAMC Annex, Maryland Exercise and Robotics Center of Excellence, 209 W. Fayette St, Rm 207, Baltimore, MD 21201; 410-605-7000, ext 3246.
}

Email: Ronald.Goodman@va.gov

http://dx.doi.org/10.1682/JRRD.2013.02.0050 
locomotor training programs can improve gait velocity and elicit changes in cortical and subcortical neural networks associated with paretic LL movements [18-21]. Efforts to improve upon treadmill-based approaches have evolved to include programmable electromechanical devices such as robotic exoskeletons to enhance locomotor therapy. As highlighted in a Cochrane review [15], there are potential benefits from using LL robotics poststroke, but we have much to learn about optimal interventions. Robotic devices can provide a useful platform for assessing comparative effectiveness of different motor learning strategies by providing versatile, interactive, task-specific training; a capacity to integrate or reward performance feedback; and the ability to precisely measure the rate and magnitude of key descriptors of motor-performance variables [10-12,1415,20-21]. In the past several years, the Baltimore Department of Veterans Affairs (VA) Medical Center, in collaboration with the Massachusetts Institute of Technology, has developed and deployed in the clinic an impedancecontrolled, modular, 2 degree of freedom (DOF)-actuated ankle robot exoskeleton (anklebot, Interactive Motion Technologies; Watertown, Massachusetts), to improve walking and balance functions poststroke by means of increasing the paretic ankle contribution during taskoriented functional activities. The rationale to focus on the ankle was due to the critical role it plays in the biomechanics of gait and balance, including providing the bulk of mechanical power needed for forward propulsion during late stance, ground clearance during swing, and shock absorption during foot strike. In addition, the ankle also plays a crucial role in the maintenance of stable upright posture, during both quiet standing and locomotion [2223]. The anklebot allows movement at the ankle in all three DOFs but provides active assistance (or resistance) in two of those DOFs, i.e., dorsiflexion (DF)/plantar flexion (PF) and inversion/eversion. It is highly backdrivable (i.e., the robot can be programmed to "get out of the way" when needed) and has low static friction, and its impedance control enables assistance "as needed" based on user deficit severity and performance during task execution. This makes the anklebot a minimally intrusive device in that it does not constrain but rather promotes volitional movement, which has been shown to be an essential component of motor learning [8,20-21]. Moreover, the anklebot is designed for use in multiple therapeutic settings (seated, upright, supine) and its 2-DOF actuation facilitates tailoring of therapies to a wide range of functional activities (e.g., seated, isolated ankle movement therapy vs tread- mill, or overground-based gait therapy). These features and the rich array of sensors (rotary and linear encoders and current sensors for precise measurement of ankle kinematics and kinetics, respectively) make the anklebot an ideal motor-learning platform as well as a precise clinical measurement instrument. Thus, our choice of using the anklebot for this study was based in part on the flexible, interactive platform it affords for implementing motorlearning paradigms and its ability to assay the temporal profile of motor performance across training. For example, our prior studies have shown that changes in passive ankle stiffness in the inversion-eversion plane to be a strong predictor of improvements in independent floor-walking speed in patients with chronic stroke [12], Similarly, movement smoothness (characterized by jerk normalized to peak movement speed) has been used to characterize motor recovery for both the upper limb and LL [10-11,2426]. Notably and relevant to this study, seated anklebot training allows for the collection of viable electroencephalography (EEG) signal with minimal movement artifact, thus fostering study of the cortical dynamics associated with motor learning.

A fundamental tenet in the field of cognitive neuroscience is that reward provides motivational incentive that elicits brain states conducive to adaptive learning [27-40]. Moreover, clinicians widely acknowledge the essential role of reward and motivation in successful rehabilitation and recovery [41]. In this regard, recent investigations have highlighted the "state of the learner" as a crucial component in learning; these studies suggest that factors including attentional focus, perceived control, and perceived reward prompt brain states in which the individual's ability to learn is enhanced [27,32,3436,38-39,42-44]. Yet we are aware of no studies in stroke in which reward has been manipulated for the purpose of increasing motor learning and recovery.

The measurement precision of the anklebot coupled with high-temporal resolution EEG provides a window to the psychophysics and neural plasticity associated with motor learning. Notably, motor learning and human motor performance studies employing EEG have robustly observed a streamlining of cortical processes as a function of learning and/or superior performance. Specifically, they observed reductions in activation (i.e., increased alpha power and/or decreased beta or gamma power) of taskirrelevant areas as well as reduced networking (EEG coherence) between motor planning and left temporal verbal association areas. During the execution of a cognitive 
motor task, the left temporal region has been implicated as necessary in the early stages of learning while progressively becoming less essential as one acquires competency. In this regard, the magnitude of motor learning would be reflected by decreased activation in and networking with this area [43-45].

In the present study, we used the impedancecontrolled anklebot as a test platform to determine whether LL robotic training would be enhanced with overt rewards and augmented feedback as compared with simply performing the same amounts of anklebot training without these reinforcements. To evaluate cortical electrophysiology, we use EEG because of its high temporal resolution and compatibility for on-task use with seated robotic training. Among the unknowns we will explore is whether LL robotic training can mediate adaptive learning responses and associated plasticity and whether putative modulators of motor learning such as reward can enhance the trainings' therapeutic efficacy or temporal response profile compared with a matched low reward (LR) group that receives the same dose of robotics training without the enhanced reward component. We predict that after nine sessions of anklebot training, subjects randomized to the high reward (HR) group will show greater improvements in paretic ankle motor control and functional gains in overground walking than those in the LR group. We further predict that, along with the increased performance and learning, we will observe concurrent changes in cortical dynamics. Specifically in the HR as compared with the LR group, we expect greater decreases (i.e., streamlining) in (1) coherence between task-relevant visuomotor networks and (2) activation in beta and gamma power in the left hemisphere verbalassociation areas that are task irrelevant (specifically electrode T7) [45-47].

\section{METHODS}

\section{Subjects}

Twenty-seven volunteers with chronic hemiparetic stroke were recruited to participate in a $3 \mathrm{wk}$, three sessions per week training program with the anklebot. Individuals with stroke who had completed all physical and occupational therapy and were not receiving any other formal rehabilitation were selected in order to isolate the effects of robotics-assisted motor learning while manipulating reward from any ongoing physical or occupational therapy and/or heterogeneous natural neural recovery. All participants provided informed consent approved by the University of Maryland School of Medicine Baltimore Institutional Review Board and the Baltimore VA Research and Development Committee. After giving informed consent, participants underwent medical screening, neurological examination, and a review of medical records prior to enrollment. Inclusion criteria were (1) index stroke $>3$ mo prior in women or men aged 21 to $85 \mathrm{yr}$; (2) residual hemiparetic gait deficits, with observable asymmetry [48]; (3) completion of all conventional physical therapy; (4) adequate language and neurocognitive function to participate in testing and training and to give informed consent; and (5) paretic DF manual muscle test score of $\geq 2$ of 5 , gravity neutral. Exclusion criteria were (1) clinical history of orthopedic, chronic pain, or neuromuscular disorders restricting participation in a short-term ankle movement training; (2) severe or global aphasia that confounds reliable testing and training; (3) Mini-Mental Status Examination score of $<23$; or (4) Center for Epidemiologic Studies Depression Scale score of $>16$ and/or untreated major depression.

\section{Anklebot Training}

Anklebot setup and training protocols were similar to those used in our prior studies [10-11]. Participants played a seated video game by moving the ankle in DF and $\mathrm{PF}$ ranges to control a cursor through gates that moved across the screen at different vertical levels (Figure 1). The primary instruction to subjects was to move the cursor to successfully pass through the gates, which appeared in a predetermined sequence located at 80 and 40 percent of each participant's volitional PF to DF excursion (defined as angular displacement of the paretic ankle from maximum PF through maximum DF). Each session included six training blocks with 60 gate targets per block. Robotic assistance was provided under a performance-based approach that used an impedance controller $[10-12,14]$ to generate torques toward the target only as needed, encouraging participants to reach targets on their own if they were able. The anklebot sensors track performance, and if the subject does not initiate the movement toward the target within $2 \mathrm{~s}$ of its appearance, the impedance controller actuates the anklebot to provide assistance, although not sufficiently to passively move the ankle to the target. The magnitude of robotic assistance for a given trial is adjusted by changing the impedance controller parameters, providing greater assistance 


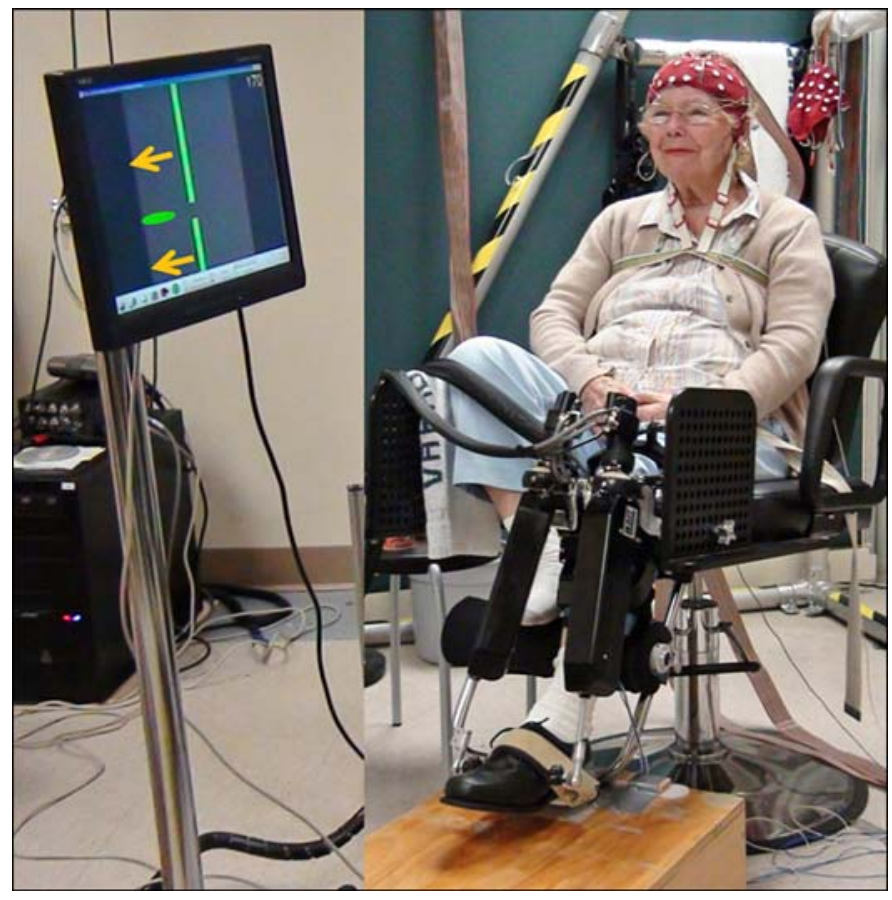

Figure 1.

Experimental setup of anklebot training with electroencephalography (EEG) monitoring. Each subject was fitted with 64-channel EEG cap and seated with anklebot mounted proximally onto fixed plate and affected leg resting at $45^{\circ}$ on padded support. Robot attached distally to customized shoe with mounting brackets for medial and lateral actuators. Event markers generated by anklebot were used to synchronize anklebot and EEG data streams. Video game required subjects to move onscreen cursor by dorsiflexing or plantar flexing their paretic ankle. Goal was to successfully maneuver through vertical gates approaching across screen from left to right (yellow arrows).

if the cursor is farther away from the target (Figure 1). All participants had their level of assist decreased at least twice and had their target ranges increased at least once over the course of the intervention. In addition, daily assessments of volitional excursion were taken in order to scale the targets on the screen presentation to correspond with each participant's volitional excursion. This was done via a scaling factor that characterized the mapping of the ankle angular displacement (degrees) to cursor movement on the screen (pixels). The scaling factor therefore may be considered as a proxy of volitional excursion, because it represents a transformation of the participants' excursion to the cursor displacement, with lower scaling values representing greater volitional excursion. The scaling factor decreased or stayed the same for all participants (pretreatment [mean \pm standard deviation]: $1.21 \pm 0.52$, posttreatment: $1.06 \pm 0.54$ ). The six blocks of assist-as-needed training were preceded and followed by two assessment blocks of 30 gates without any robotic assistance to assess independent performance. Thus, each session amassed a total of 480 targeted movements and lasted $\sim 1 \mathrm{~h}$.

Participants were randomly assigned to receive high or low levels of monetary reward, social interaction, and performance feedback. HR subjects received verbal encouragement, a running display of their score, access to past scores, and eligibility for immediate (within-session) and long-term (study-wide "grand competition") monetary prizes. Verbal encouragement was given only at the end of each of the six training blocks (e.g., "Way to go, you had to work really hard to get those last two gates, and you did it!"). Within-session monetary rewards were based on the individual participant's performance (i.e., if they beat their score from the previous block, they won \$5). However, in order to ensure that participants kept striving for optimal performances, we told them that we were also considering factors other than their score, such as movement smoothness (i.e., measures derived from robotic recordings). To make this believable, researchers were instructed to withhold a reward for an improved score once a session. Monetary rewards were awarded to the HR group at the end of each winning block, receiving between $\$ 0$ and $\$ 25$ per session for all nine sessions. The LR group received sparse but controlled social interaction without verbal encouragement, scoring feedback, or prizes; although naïve to the information prior to or during training, they did receive equivalent monetary compensation at the conclusion of their last day of training.

\section{Assessments}

Participants were assessed at the beginning and completion of training with clinical and robot-derived measures to evaluate ankle motor control and gait function. EEG was collected during all unassisted and assisted trials on day 1 (pretest) and day 9 (posttest).

\section{Motor Behavior}

\section{Motor Control Measures}

Robot-based metrics were calculated from positional data recorded $(200 \mathrm{~Hz})$ during the unassisted trials. These included averages for the percentage of successful gate 
passages, peak and mean angular velocity, and normalized jerk. Movement velocity and acceleration were obtained from the first and second derivatives of the position time series; the derived profiles were rectified and used to calculate mean and peak velocity. A movement or submovement was considered to have begun and terminated when the angular velocity first rose above and then dropped below 2 percent of the peak velocity. Movement smoothness was characterized by normalized jerk, the first derivative of acceleration, divided by the peak velocity, so as to not confound smoothness with movement speed.

\section{Learning Rate}

To determine whether reward influences the rate of motor skill acquisition, we assessed the learning rate using a best-fit approach to determine the time-related changes in key motor control indices during unassisted trials across the nine training days. Both logarithmic power models and linear regression were evaluated, with the linear approach yielding a better statistical fit than the logarithmic power models. Thus, for each individual, a simple regression was conducted between time (session) and the motor control outcomes. If the two variables were significantly related, the slope (beta coefficient) was entered as the dependent measure for the group contrasts. Otherwise, if the regression failed to reach statistical significance (i.e., $p>0.05$, indicating no significant relationship between the two variables and hence no learning), then a value of 0 was used. This analysis allowed for a comparison of the learning rates between the two groups.

\section{Gait Function}

Independent walking function was assessed by having subjects warm up by walking across an instrumented walkway two times at their preferred speed with 5 min rests after each trial. Subjects then performed two trials in which they were instructed to walk "as safely and fast as possible" across the same $8 \mathrm{~m}$ instrumented walkway (CIR Systems Inc/GAITRite; Sparta, New Jersey) that sampled at $100 \mathrm{~Hz}$ with at least two steps taken before the start and after the end to eliminate acceleration and deceleration phases. Partial foot contacts at the extremes of the recording area were removed as needed. Spatiotemporal outcomes included mean velocity (centimeters per second), stride length (centimeters), cadence (steps per minute), and relative paretic single-support and double- support durations (percent cycle); double-support measurements were calculated using combined phases.

\section{Psychophysiological Recordings and Processing}

The EEG measures of spectral power and coherence were assessed for all unassisted and assisted trials on day 1 (pretest) and day 9 (posttest). EEG data were collected (actiCap system, Brain Products GmbH; Munich, Germany) from 64 sites, labeled in accordance with an extended 10 to 20 international system [49]. The EEG data were online referenced to the right earlobe with a common ground at the $\mathrm{FPz}$ site. Electrode impedances were maintained below $10 \mathrm{k} \Omega$ with bandpass filters set at 0.01 to $100 \mathrm{~Hz}$ with a sampling rate of $1 \mathrm{kHz}$. The EEG signal was digitized using a BrainAmp DC amplifier (Brain Products $\mathrm{GmbH}$ ) linked to BrainVision Recorder software version 1.10 (Brain Products $\mathrm{GmbH}$ ). All signal processing of the EEG data was conducted using BrainVision Analyzer software version 2.0 (Brain Products $\mathrm{GmbH}$ ). Continuous data from each unassisted trial were offline referenced to an averaged ears montage and then low-pass filtered at $55 \mathrm{~Hz}$ with a $48 \mathrm{~dB}$ roll-off and band-stopped between 55 and $65 \mathrm{~Hz}$ using a zero-phase Butterworth filter. Next, all sweeps were visually inspected, and trials containing nonstereotyped artifacts were excluded from further analyses, a technique referred to as pruning, which improves the ability of an independent component analysis (ICA) to identify stable components [50]. Eye movement artifact was reduced using the ICA-based ocular artifact rejection function within the BrainVision Analyzer software version 1.10 (Brain Products $\mathrm{GmbH}$ ); electrode FP2 served as the vertical electro-oculogram channel and electrodes AF7 and AF8 served as the bipolar horizontal electro-oculogram channel. The vertical electro-oculogram algorithm searches for an eye-blink template in channel FP2 and then finds ICAderived components that account for a user-specified amount of variance $(70 \%)$ in the template-matched portion of the signal from electrode FP2. The horizontal electrooculogram algorithm finds ICA-derived components that account for a user-specified amount of variance (30\%) in the entire signal from the horizontal electro-oculogram channel (bipolar electrodes AF7 and AF8). These components were removed from the raw EEG signal, and the recording was reconstructed for further processing.

Data from each unassisted condition were epoched into $1 \mathrm{~s}$ sweeps. These epochs were baseline corrected using the entire sweep and then visually inspected to remove any remaining sweeps that contained artifact or 
amplitudes of more than $75 \mu \mathrm{V}^{2}$. Epochs were then transformed using the discrete Fourier transform, employing a Hamming window with a 50 percent overlap. Averages of spectral power (microvolts squared) were calculated across $1 \mathrm{~Hz}$ bins and averaged across the frequency bandwidths: theta $(3-8 \mathrm{~Hz})$, low alpha $(8-10 \mathrm{~Hz})$, high alpha $(10-13 \mathrm{~Hz})$, beta $(13-30 \mathrm{~Hz})$, and gamma $(30-50 \mathrm{~Hz})$. These averages were then natural log-transformed prior to statistical analysis.

The same artifact-reduced epochs were used to compute coherence using the cross- and autospectral densities, i.e., (Equations (1)-(2)):

$$
\operatorname{Coh}\left(c_{1}, c_{2}\right)(f)=\left|\operatorname{Cov}\left(c_{1}, c_{2}\right)(f)\right|^{2} /\left(\operatorname{Cov}\left(c_{1}, c_{1}\right)(f)|\cdot| \operatorname{Cov}\left(c_{2}, c_{2}\right)(f) \mid\right)(\mathbf{1})
$$

and

$$
\operatorname{Cov}\left(c_{1}, c_{2}\right)(f)=\sum_{i}\left(c_{1, i}(f)-\bar{c}_{1}(f)\right) \cdot\left(c_{2, i}(f)-\bar{c}_{2}(f)\right),
$$

where $\operatorname{Coh}()=$ spectral coherence function; $c_{1}$ and $c_{2}=$ signals from the electrode pair and their sample means, respectively; $f=$ frequency; and $\operatorname{Cov}()=$ covariance function that is computed across $1 \mathrm{~Hz}$ bins, then averaged across the frequency theta $(3-8 \mathrm{~Hz})$, alpha $(8-13 \mathrm{~Hz})$, low beta $(13-20 \mathrm{~Hz})$, high beta $(20-30 \mathrm{~Hz})$, and gamma (30$50 \mathrm{~Hz}$ ) bandwidths. Specifically, coherence was computed between electrode $\mathrm{Fz}$, which overlies the motor planning region and the following electrodes: F3, F4, C3, C4, T7, $\mathrm{T} 8, \mathrm{P} 3, \mathrm{P} 4, \mathrm{O} 1$, and $\mathrm{O} 2$, and between frontoparietal electrodes: F3-P3/P4 and F4-P3/P4. All coherence values were subjected to a Fisher $z$-transformation prior to statistical analysis to approximate a normal distribution.

\section{Statistical Analyses}

To evaluate change across the training intervention, 2 (group) $\times 2$ (time) mixed-design repeated-measures analyses of variance (ANOVAs) were conducted on the anklebotderived motor control measures. Post hoc analyses were conducted using paired $t$-tests. Spectral and coherence measures were subjected to separate 2 (group) $\times 2$ (time) $\times$ 2 (hemisphere) $\times 5$ (region) mixed-design repeatedmeasures ANOVAs for each of the frequency bands. Interactions involving group and time with $p \leq 0.10$ were examined by running secondary repeated-measures ANOVAs separated by group. Post hoc analyses were computed using the Tukey test. The frontoparietal coherence measures were subjected to separate 2 (group) $\times 2$ (time) $\times$ 2 (region-frontal) $\times 2$ (region-parietal) mixed-design repeated-measures ANOVAs and were then subjected to the same analysis as the coherence measures described previously. Group differences from the learning rate data were subjected to separate independent $t$-tests for each of the anklebot-derived variables. In light of the group differences in EEG and motor control, a secondary analysis using paired $t$-tests separately for each group was performed on spatiotemporal gait parameters. Significance levels were set at $p \leq 0.05$.

\section{RESULTS}

\section{Participants}

Twenty-seven participants were recruited; seventeen were enrolled. Seven withdrew due to (1) illness unrelated to study (2 participants), (2) failure to comply with testing and training schedule (1 participant), (3) return to physical therapy (1 participant), (4) a new job that did not allow subject to get to laboratory during hours of operation (1 participant), (5) inability to recontact (1 participant), and (6) relocation (1 participant). Table 1 presents participants' clinical and demographic information. Independent $t$-tests at baseline revealed no differences between groups with regard to age or time since stroke, and the use of assistive devices was comparable between groups. Also, the groups did not differ on any of the motor control variables (mean velocity, peak velocity, successful gate passages, and normalized jerk) or any of the gait variables, including velocity, stride length, cadence, and relative paretic single-support and double-support times (Table 2).

\section{Motor Behavior}

\section{Motor Control}

Smoothness measures differed between groups over time, because normalized jerk had a significant $2 \times 2$ interaction $(F(1,8)=9.63, p=0.02)$. Post hoc analysis revealed that the HR group significantly reduced jerk as a function of time $(t(4)=3.05, p=0.04)$, whereas the LR group was relatively unchanged (see exemplar tracings, Figure 2). The percent of successful passages revealed a main effect for time such that both groups were more successful in gate passages posttraining relative to pretraining 
Table 1.

Patient demographics for each of two groups. Independent $t$-tests revealed that groups were not different with regard to age, time poststroke, and baseline gait velocity $(p>0.10)$.

\begin{tabular}{|c|c|c|c|c|c|c|c|}
\hline Group & Sex & $\begin{array}{l}\text { Age } \\
(\text { yr) }\end{array}$ & $\begin{array}{c}\text { Time } \\
\text { Poststroke } \\
\text { (mo) }\end{array}$ & Lesion Location & $\begin{array}{l}\text { Affected } \\
\text { Side }\end{array}$ & Assistive Device & $\begin{array}{l}\text { Baseline Gait } \\
\text { Velocity }(\mathrm{cm} / \mathrm{s})\end{array}$ \\
\hline \multirow[t]{5}{*}{$\begin{array}{l}\text { High } \\
\text { Reward }\end{array}$} & $\mathrm{F}$ & 47 & 9 & $\begin{array}{l}\text { Right middle cerebral artery affecting } \\
\text { right frontal, temporal, and parietal } \\
\text { lobes and basal ganglia. }\end{array}$ & $\mathrm{L}$ & AFO, Quad-Point Cane & 30.8 \\
\hline & $\mathrm{F}$ & 58 & 27 & Right cerebellar. & $\mathrm{R}$ & Walker & 81.3 \\
\hline & $\mathrm{F}$ & 82 & 152 & Right cerebral infarct at internal capsule. & $\mathrm{L}$ & Single-Point Cane & 52.0 \\
\hline & M & 62 & 77 & Left basal ganglia. & $\mathrm{L}$ & Single-Point Cane & 134.6 \\
\hline & M & 62 & 61 & $\begin{array}{l}\text { Transient ischemic attack of right internal } \\
\text { carotid artery. }\end{array}$ & $\mathrm{L}$ & AFO & 95.7 \\
\hline \multirow[t]{5}{*}{$\begin{array}{l}\text { Low } \\
\text { Reward }\end{array}$} & $\mathrm{F}$ & 47 & 103 & $\begin{array}{l}\text { Right middle cerebral artery and right } \\
\text { anterior cerebral artery. }\end{array}$ & $\mathrm{L}$ & AFO, Single-Point Cane & 64.4 \\
\hline & $\mathrm{F}$ & 64 & 107 & $\begin{array}{l}\text { Right middle cerebral artery (ischemic) } \\
\text { and right basal ganglia (hemorrhagic). }\end{array}$ & $\mathrm{L}$ & AFO, Single-Point Cane & 37.6 \\
\hline & M & 42 & 203 & Right intracranial hematoma. & $\mathrm{L}$ & $\mathrm{AFO}$ & 122.8 \\
\hline & M & 45 & 330 & Right temporal parietal junction. & $\mathrm{L}$ & $\mathrm{AFO}$ & 133.1 \\
\hline & M & 76 & 33 & Right small medullary. & $\mathrm{L}$ & Wheelchair & $\begin{array}{l}\text { Excluded from } \\
\text { gait analysis. }\end{array}$ \\
\hline
\end{tabular}

Table 2.

Motor behavior variables before and after anklebot training intervention. Pre- and posttreatment values are separated by group. Additionally, percent change is displayed.

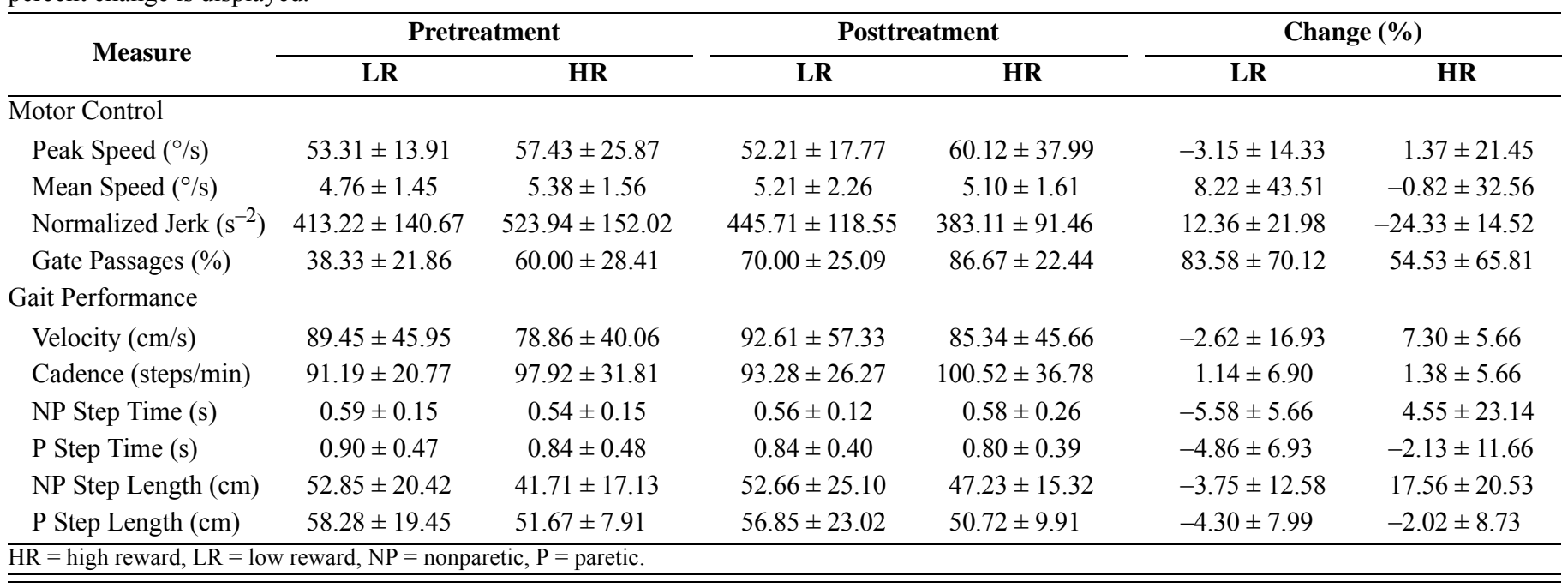

$(F(1,6)=60.25, p<0.001)$. Mean and peak velocities were not significantly different over time (Table 2).

\section{Learning Rates}

Across the course of training, the level of assist was progressively decreased based on performance success (defined as whether or not the participant was successful in attaining at least $80 \%$ of the total targets); however, all measurements used in the learning rate analysis were based on unassisted trials taken just prior to training. Analysis of normalized jerk revealed faster gains in smoothness by the HR group relative to the LR group $(t(8)=2.54, p=0.03$ ) 

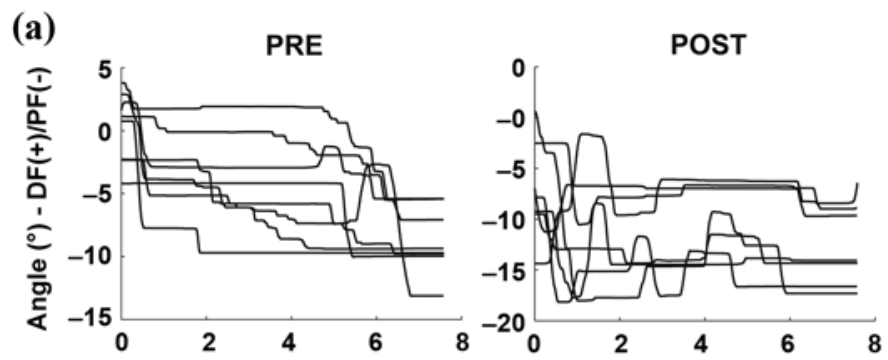

(b)
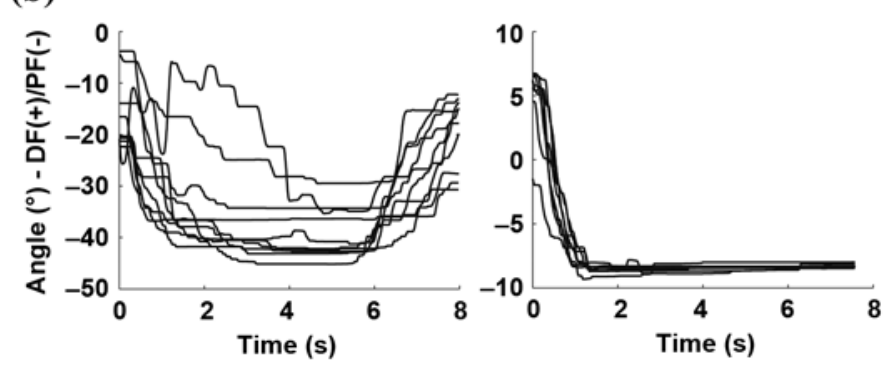

Figure 2.

Ensemble plots of unassisted plantar flexion (PF) movements made by exemplar subjects in (a) low reward and (b) high reward (HR) groups, measured at baseline (pre) and completion (post). Comparison of pre- versus post-HR tracings demonstrates improvement in key features of volitional ankle motor control (lower end-point variability reflects greater accuracy, greater slopes reflect higher speed to target, and more "tightly bounded" traces reflect greater smoothness). DF = dorsiflexion.

(Table 2). The other metrics of ankle motor control (mean velocity, peak velocity, and number of successful gate passages) were not significantly different between groups. Analysis of normalized jerk revealed faster gains in smoothness by the HR group relative to the LR group $(t(8)=2.54, p=0.03)$ (Figure 3).

The learning rate in this short intervention may not have plateaued, and thus, the better fit exhibited by linear regression as compared with logarithmic power models (typically the better fit for rates of motor learning) is consistent with the linear portion (pre-plateau) of the logarithmic power model curve. We chose the 3 wk, 9-session length of this intervention after analyzing the results of a $6 \mathrm{wk}, 18$-session anklebot feasibility study in which many participants began to plateau at $3 \mathrm{wk}$. In this regard, the changes observed in floor walking velocity represent slightly more than half the improvement observed in a 6 wk study [13].

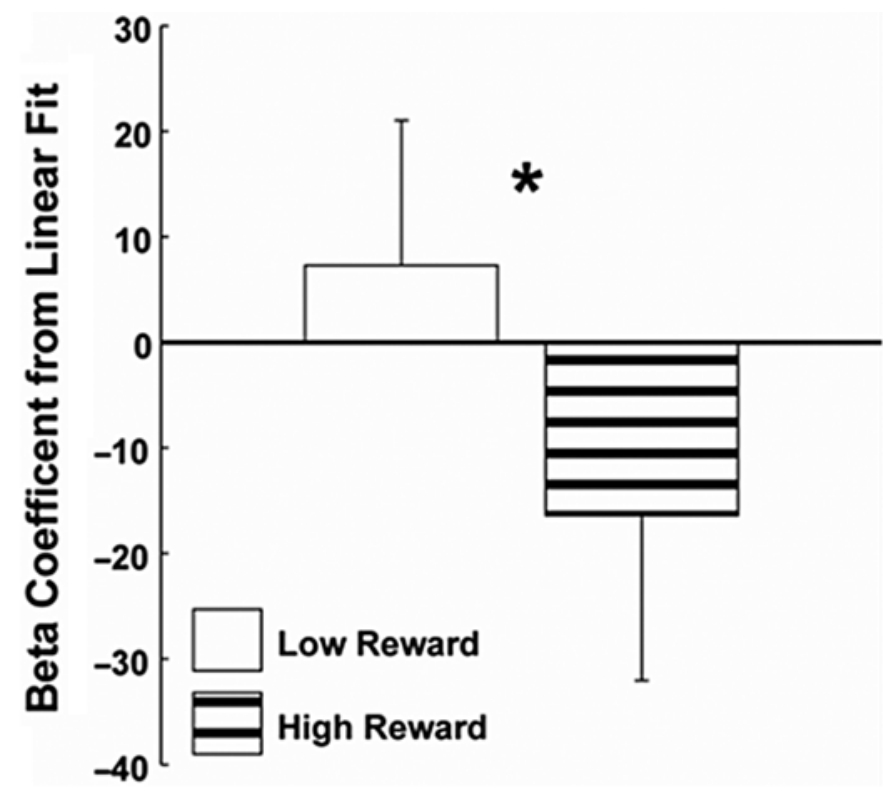

Reward

Figure 3.

Learning rate of motor control. Mean beta coefficient (slope/ learning rate) by group, averaged across training. Lower values of jerk represents smoother movements to target. Note: Although it looks as if low reward group's movements are getting more jerky, relationship is not significant (as per $2 \times 2$ analysis of variance results) and as such should be considered as relatively stable performance across time. ${ }^{*} p<0.05$.

\section{Gait Function}

Group differences were revealed during the analysis of the fast floor walking condition. The HR group had a significant increase in nonparetic step length $(t(7)=2.69$, $p=0.05)$. The other spatiotemporal outcomes did not reveal any significant changes over the 3 wk (Table 2).

\section{Electroencephalograpy Measures}

\section{Spectral Power}

Based on a priori notions of the negative role of the left temporal region in motor planning, data in both the beta and gamma bandwidths were subjected to $t$-tests [45-47]. The HR group showed a significant decrease in left temporal gamma power in posttraining relative to

\footnotetext{
* One participant in the LR group was excluded from all functional gait analyses because he was unable to engage in independent walking.
} 
baseline $(t(1,4)=2.73, p=0.05)$. Theta, low alpha, high alpha, beta, and gamma: no bandwidths showed significant findings that included both time and group.

\section{Coherence to Motor Planning}

Theta. A group $\times$ time $\times$ hemisphere interaction was observed $(F(1,8)=6.10, p=0.04)$. Separate within-group post hoc analyses revealed a time $\times$ hemisphere interaction for the LR group $(F(1,4)=67.38, p=0.01)$. Post hoc testing revealed a significant increase in theta coherence between the left hemisphere and motor planning region $(p<0.01)$.

Alpha. A group $\times$ time $\times$ hemisphere interaction was observed $(F(1,8)=4.84, p=0.06)$. Post hoc analysis examining the two groups separately revealed a time $\times$ hemisphere interaction for the LR group $(F(1,4)=8.27$, $p=0.05)$, but post hoc tests revealed no meaningful differences.

Low beta. A group $\times$ time $\times$ hemisphere interaction was observed $(F(1,8)=3.40, p=0.10)$. Post hoc analysis examining the two groups separately revealed a time $\times$ hemisphere interaction for the LR group $(F(1,4)=16.30$, $p=0.02)$. Post hoc testing revealed a significant decrease in low beta coherence between the right hemisphere and motor planning region $(p<0.01)$ (Figure 4).

\section{Frontoparietal Coherence}

Theta. A group $\times$ time $\times$ region-frontal interaction was observed $(F(1,8)=5.47, p=0.05)$. Post hoc analysis examining the two groups separately revealed a time $\times$ region-frontal interaction for the HR group $(F(1,4)=$ $17.93, p=0.02)$. Post hoc testing revealed a significant decrease in theta coherence between the left frontal region and bilateral parietal regions $(p<0.01)$.

Low beta. A group $\times$ time $\times$ region-frontal interaction was observed $(F(1,8)=6.50, p=0.03)$. Post hoc analysis examining the two groups separately revealed a time $\times$ region-frontal interaction for the HR group $(F(1,4)=8.98, p=0.04)$. Post hoc testing revealed a significant decrease in low beta coherence between the left frontal region and bilateral parietal regions $(p<0.01)$ (Figure 4).

\section{DISCUSSION}

The current study shows that reward-based ankle robotics training improved both the rate of motor learning
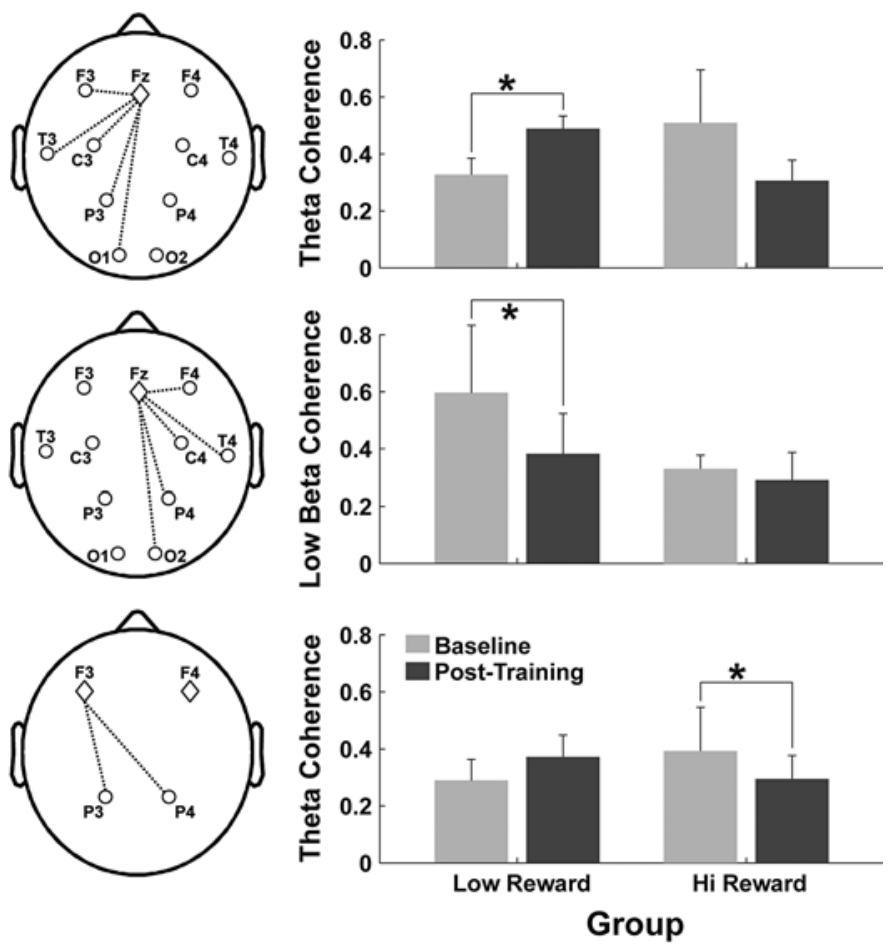

Figure 4.

Spectral coherence results from Tukey post hoc test of results from secondary, separated by group analyses of variance (2 [time] $\times 2$ [hemisphere]). Left panels display scalp montages corresponding to change in coherence values from baseline to posttraining displayed in right panels. Specific bandwidths within which interactions occurred are displayed between panels. ${ }^{*} p<0.01$.

and the motor control of paretic ankle movements in persons with chronic hemiparetic stroke. Specifically, compared with the LR group, the HR group showed significantly faster learning rates and a greater increase in trajectory smoothness, as measured by normalized jerk.

Notably, the emergence of movement smoothness as a key mechanistic descriptor of motor performance is consistent with similar findings resulting from arm robot therapy [24-25,51]. A common finding was that movement smoothness improved through the blending of discrete episodic submovements resulting in a more continuous motion. It is not yet known whether a similar process occurs at the LL; however, preliminary observations from anklebot training across different phases of stroke (subacute and chronic) further support the notion that changes in smoothness may be an important indicator of motor recovery. In this regard, minimization of jerk during 
goal-oriented movements may be a unifying mechanism deployed by the central nervous system (CNS).

Additionally, as compared with the dose-matched LR group, the HR group exhibited gains in nonparetic step length (suggestive of greater paretic foot push-off). While these gains were relatively modest as compared with more intensive and/or longer-term treadmill or overground gait training paradigms, this relatively rapid change in gait was observed after only nine sessions of intensive robotic training and more importantly, from a non-task-specific paradigm (i.e., we did not actually train gait function). These results further support earlier findings that improving underlying motor control and reducing impairments through seated anklebot therapy translates into positive changes in key spatial-temporal aspects of gait patterning [10-12]. Important to the purpose of this study, these results suggest that adding reward and augmented feedback to LL robotics may accelerate the acquisition of these gains.

Notably, the HR group significantly decreased frontoparietal coherence in the contralesional hemisphere and decreased activation in the left temporal verbal association area. These changes in cortical dynamics suggest that reward-induced neural plasticity may have modulated the state of the learner in an adaptive manner, facilitating responses beyond that of unrewarded anklebot training [27,35,37-38,43]. Taken together, our results provide the first evidence that using overt rewards modulate the state of learner during robotics-assisted rehabilitation can enhance functionally relevant LL motor learning and that increased reward facilitates adaptive neuroplasticity in the chronic-hemiparetic brain.

Although current principles of cognitive motor learning recognize a positive relationship between reward and motor-learning trajectory, empirical evidence in stroke rehabilitation and recovery is sparse. This is the first electrophysiological investigation of robotics training in people with hemiparetic stroke to demonstrate that reward enhances motor learning and suggests that, in general, it may deserve a more prominent role in designing stroke therapies. That the improved motor control and independent floor walking of the HR group was associated with group differences in EEG suggests that the reward manipulation primed the cognitive motor-learning circuitry to mediate these improvements. Furthermore, these convergent changes provide additional evidence that cortical processes play an integral role in LL motor learning and possibly even more so in a patient popula- tion that needs to exert more conscious effort for the otherwise largely automatic act of walking.

Although we were not able to directly measure dopamine and norepinephrine, our conceptual framework for investigating the effects of reward manipulation was based on the established role they play in functional neuroanatomy and learning. It is well known that the elicitation of emotion in a laboratory setting is challenging. Thus, in order to ensure that we would evoke a high enough level of affective response to observe a difference between the two groups, we combined and delivered three forms of reward (monetary, performance feedback, and social encouragement) [52]. In this regard, the combination of reward modalities may be thought of as providing an enriched learning environment that fosters increased attention and engagement during this repetitive task, both of which have been associated with augmented learning [30-31,34-35,53-55]. Further, there is substantial support for the notion that emotion and its corresponding circuitry largely determine the goals of human behavior (but not the behavior itself) [53-54]. The affective areas underlying reward-based modulations (i.e., positive affect) have been associated with increased arousal and attention, both of which have been linked to enhanced neural plasticity and subsequent learning [29,31,33-44,55]. These networks provide the functional neuroanatomy to initiate the autonomic and neuromodulatory increases that augment learning [27-31,33-35,39-40,53-55]. Hence, reward may enhance the positive effects of modular robotic therapies by promoting CNS plasticity. Further studies are needed to determine the neurochemistry underlying this plasticity and how reward can best be administered with respect to delivery modalities, level, and timing profiles of rewardbased feedback to improve neuromotor and functional outcomes.

The stroke literature reports widespread heterogeneity in lesion location, symptomology, and treatment response [21,56-57]. However, a number of studies posit an association between increases in contralesional brain activity and reduced recovery, suggesting that increased inhibitory signaling from contralesional motor cortices to the relevant ipsilesional motor regions interferes with control of the affected limb [21,56-57]. Likewise, we observed improved motor behavior in conjunction with decreases in frontoparietal networking (i.e., decreased EEG coherence) in the contralesional hemisphere for the HR group only. In contrast, the slower learning in the LR group was accompanied by an increased reliance on 
contralesional but not ipsilesional areas for motor planning and execution, suggesting that the rewards may have influenced the decrease in contralesional inhibition observed in the HR group. Within the framework of this theoretical model, the increased contralesional and decreased ipsilesional networking observed in the LR group suggest they may have engaged a neural network (strategy) that, although seemingly useful in the context of the LR anklebot training received in this study, appears to limit a person with stroke's ability to optimize recovery of function. Additionally, the HR group's decreased gamma power in the left temporal verbal-association area is consistent with the nondisabled motor performance literature, which suggests that reduced activity in taskirrelevant areas (left hemisphere verbal association region) is associated with increased performance [4547]. As such, this decreased activation and the streamlined contralesional coherence may have influenced or reflected the faster rate of learning observed in the HR group. This notion finds support in the multistage motor learning theory of Fitts and Posner, which posits a higher level of verbal input in the early stages of learning followed by two subsequent stages of learning in which deliberate practice results in motor processes that become more refined in the second stage and eventually automatic in the third [58].

Anklebot training is a performance-based experience that seeks to increase paretic ankle contributions in walking and balance control by priming the neuromotor system through principles of motor learning, including high volumes of repetition, performance feedback via a video game-based format, goal setting, and reduced reliance on robotic assistance to complete prescribed movements [10$12,14]$. The power of virtual reality and video game-based therapies has shown promise in augmenting motor recovery [59-61]. Coupling these technologies with classic reward conditioning optimized through principles of clinical and cognitive motor-neuroscience may prime CNS plasticity, enhance the state of the learner, and increase the rate and eventual level of recovery in people with stroke. Our findings suggest that engagement of reward circuitry during highly structured, robotics-assisted, motor learningbased therapies appears to be a promising adjunct to neuromotor practice alone in people with stroke.

Although monetary rewards are likely not feasible in a clinical setting, the primary conceptual aim of this study was to engage reward circuitry (affective networks) by positively changing the state of the learner. While potentially more relevant to the clinic, both social interaction and performance feedback were employed in this study and offer a viable platform for development of successful reward-based rehabilitation. As mentioned previously, manipulations such as perceived reward, perceived control, level of challenge, meditation, and aerobic exercise all have exhibited the capability of positively influencing learning and/or performance through modulations of the state of the learner [27-39,61-62]. In this regard, the current study employs monetary reward as but one of many possible motivational influences capable of enhancing the state of the learner in order to improve recovery (learning) in a clinical setting.

Despite the small sample size, the study's results underscore the robustness of the HR treatment effect, and while the EEG findings were not causally linked to the gains in motor control and overground walking, they provide plausible neurophysiological evidence for a statedependent increase in activity-dependent plasticity associated with specific improvement in a precisely measured motor task across one LL joint. Hence, our findings support further exploration using EEG simultaneously with robotics training to advance our understanding of CNS plasticity and to inform the design of more effective therapies to improve motor function following stroke [63-67]. However, our findings in this relatively brief robotics intervention are limited; future randomized clinical trials are needed to determine whether ankle robotics with versus without reward produces any durable, clinically meaningful mobility improvements in people with stroke.

\section{CONCLUSIONS}

In summary, we report the first evidence from a small comparative effectiveness study: that a multifaceted reward scheme can enhance the rate and degree of motor learning with ankle robotics-assisted rehabilitation. That these findings are accompanied with distinctively altered task-related cortical dynamics in the HR versus the LR group suggests that reward tied to specific motor training of the paretic ankle accelerates activity-dependent brain plasticity to improve motor control, even years after a disabling stroke. Further clinical studies are needed to determine whether reward bolsters the efficiency and depth of motor learning to enhance LL robotics-assisted motor recovery across the different time phases of stroke recovery. 


\section{ACKNOWLEDGMENTS}

\section{Author Contributions:}

Study concept and design: R. N. Goodman, L. W. Forrester, R. F. Macko. Data acquisition: J. Diaz, B. C. Jung, R. N. Goodman, J. C. Rietschel. Data processing: J. Diaz, B. C. Jung, R. N. Goodman, J. C. Rietschel, A. Roy.

Data analysis and interpretation: R. N. Goodman, J. C. Rietschel, A. Roy, L. W. Forrester.

Drafting of manuscript: R. N. Goodman, J. C. Rietschel, A. Roy,

L. W. Forrester, R. F. Macko.

Study supervision: R. F. Macko.

Financial Disclosures: The authors have declared that no competing interests exist.

Funding/Support: This material was based on work supported by the VA Rehabilitation Research and Development Service (CDA-1 grant A7251M) and by a pilot grant from the VA Maryland Exercise and Robotics Center of Excellence.

Additional Contributions: We would like to acknowledge the participants for their hard work and commitment to the study. We would also like to acknowledge the help of Megan O'Connell, Robert Asbury, and Derrick Bickel during data collection and preprocessing and Ozell Sanders during data processing.

Institutional Review: Recruitment and informed consent procedures were approved by the University of Maryland School of Medicine Baltimore Institutional Review Board (protocol HP-00043705) and the Baltimore VA Research and Development Committee.

Participant Follow-Up: The authors do not plan to inform participants of the publication of this study. Participants have met with the investigators to discuss the insights from their individual training sessions. Disclaimer: The views expressed by contributors are their own and do not necessarily reflect the official policy of the VA.

\section{REFERENCES}

1. American Heart Association [Internet]. Heart and stroke statistics 2010. Dallas (TX): AHA; 2014. Available from: http://www.heart.org/HEARTORG/General/Heart-and-StrokeAssociation\%20Statistics_UCM 319064 SubHomePage.jsp

2. Medicare.gov [Internet]. Health database. Baltimore (MD): Centers for Medicare and Medicaid Services; 2012. Available from: https://data.medicare.gov/

3. Hachinski V, Iadecola C, Petersen RC, Breteler MM, Nyenhuis DL, Black SE, Powers WJ, DeCarli C, Merino JG, Kalaria RN, Vinters HV, Holtzman DM, Rosenberg GA, Wallin A, Dichgans M, Marler JR, Leblanc GG. National Institute of Neurological Disorders and Stroke-Canadian Stroke Network vascular cognitive impairment harmonization standards. Stroke. 2006;37(9):2220-41. [PMID:16917086] http://dx.doi.org/10.1161/01.STR.0000237236.88823.47

4. Jørgensen HS, Nakayama H, Raaschou HO, Olsen TS. Recovery of walking function in stroke patients: The Copenhagen Stroke Study. Arch Phys Med Rehabil. 1995;
76(1):27-32. [PMID:7811170]

http://dx.doi.org/10.1016/S0003-9993(95)80038-7

5. Forster A, Young J. Incidence and consequences of falls due to stroke: A systematic inquiry. BMJ. 1995;311(6997): 83-86. [PMID:7613406] http://dx.doi.org/10.1136/bmj.311.6997.83

6. Dennis MS, Lo KM, McDowall M, West T. Fractures after stroke: frequency, types, and associations. Stroke. 2002; 33(3):728-34. [PMID:11872896]

http://dx.doi.org/10.1161/hs0302.103621

7. Kanis J, Oden A, Johnell O. Acute and long-term increase in fracture risk after hospitalization for stroke. Stroke. 2001;32(3):702-6. [PMID:11239190] http://dx.doi.org/10.1161/01.STR.32.3.702

8. Krakauer JW. Motor learning: Its relevance to stroke recovery and neurorehabilitation. Curr Opin Neurol. 2006;19(1): 84-90. [PMID:16415682] http://dx.doi.org/10.1097/01.wco.0000200544.29915.cc

9. Macko RF, Ivey FM, Forrester LW, Hanley D, Sorkin JD, Katzel LI, Silver KH, Goldberg AP. Treadmill exercise rehabilitation improves ambulatory function and cardiovascular fitness in patients with chronic stroke: a randomized, controlled trial. Stroke. 2005;36(10):2206-11. [PMID:16151035] http://dx.doi.org/10.1161/01.STR.0000181076.91805.89

10. Forrester LW, Roy A, Krebs HI, Macko RF. Ankle training with a robotic device improves hemiparetic gait after a stroke. Neurorehabil Neural Repair. 2011;25(4):369-77.

[PMID:21115945] http://dx.doi.org/10.1177/1545968310388291

11. Roy A, Forrester LW, Macko RF. Short-term ankle motor performance with ankle robotics training in chronic hemiparetic stroke. J Rehabil Res Dev. 2011;48(4):417-29. [PMID:21674391] http://dx.doi.org/10.1682/JRRD.2010.04.0078

12. Roy A, Forrester LW, Macko RF, Krebs HI. Changes in passive ankle stiffness and its effects on gait function in people with chronic stroke. J Rehabil Res Dev. 2013;50(4): 555-72. [PMID:23934875] http://dx.doi.org/10.1682/JRRD.2011.10.0206

13. Barbeau H, Visintin M. Optimal outcomes obtained with body-weight support combined with treadmill training in stroke subjects. Arch Phys Med Rehabil. 2003;84(10): 1458-65. [PMID:14586912] ttp://dx.doi.org/10.1016/S0003-9993(03)00361-7

14. Krebs HI, Hogan N, Aisen ML, Volpe BT. Robot-aided neurorehabilitation. IEEE Trans Rehabil Eng. 1998;6(1): 75-87. [PMID:9535526] http://dx.doi.org/10.1109/86.662623

15. Mehrholz J, Werner C, Kugler J, Pohl M. Electromechanical-assisted training for walking after stroke. Cochrane Database Syst Rev. 2007;(4):CD006185.

[PMID:17943893] 
16. Moseley AM, Stark A, Cameron ID, Pollock A. Treadmill training and body weight support for walking after stroke. Cochrane Database Syst Rev. 2003;(3):CD002840. [PMID:12917932]

17. Duncan PW, Sullivan KJ, Behrman AL, Azen SP, Wu SS, Nadeau SE, Dobkin BH, Rose DK, Tilson JK, Cen S, Hayden SK; LEAPS Investigative Team. Body-weightsupported treadmill rehabilitation after stroke. $\mathrm{N}$ Engl J Med. 2011;364(21):2026-36. [PMID:21612471] http://dx.doi.org/10.1056/NEJMoa1010790

18. Luft AR, Macko RF, Forrester LW, Villagra F, Ivey F, Sorkin JD, Whitall J, McCombe-Waller S, Katzel L, Goldberg AP, Hanley DF. Treadmill exercise activates subcortical neural networks and improves walking after stroke: A randomized controlled trial. Stroke. 2008;39(12):3341-50. [PMID:18757284] http://dx.doi.org/10.1161/STROKEAHA.108.527531

19. Luft AR, Forrester L, Macko RF, McCombe-Waller S, Whitall J, Villagra F, Hanley DF. Brain activation of lower extremity movement in chronically impaired stroke survivors. Neuroimage. 2005;26(1):184-94. [PMID:15862218] http://dx.doi.org/10.1016/j.neuroimage.2005.01.027

20. Huang VS, Krakauer JW. Robotic neurorehabilitation: A computational motor learning perspective. J Neuroeng Rehabil. 2009;6:5. [PMID:19243614] http://dx.doi.org/10.1186/1743-0003-6-5

21. Rijntjes M. Mechanisms of recovery in stroke patients with hemiparesis or aphasia: New insights, old questions and the meaning of therapies. Curr Opin Neurol. 2006;19(1):76-83. [PMID:16415681] http://dx.doi.org/10.1097/01.wco.0000203886.28068.38

22. Nashner LM, McCollum G. The organization of human postural movements: A formal basis and experimental synthesis. Behav Brain Sci. 1985;8(1):135-50. http://dx.doi.org/10.1017/S0140525X00020008

23. Winter DA. Human balance and posture control during standing and walking. Gait Posture. 1995;3(4):193-214. http://dx.doi.org/10.1016/0966-6362(96)82849-9

24. Rohrer B, Fasoli S, Krebs HI, Hughes R, Volpe B, Frontera WR, Stein J, Hogan N. Movement smoothness changes during stroke recovery. J Neurosci. 2002;22(18):8297-8304. [PMID:12223584]

25. Rohrer B. Evolution of movement smoothness and submovement patterns in persons with stroke [dissertation]. [Cambridge, MA]: Massachusetts Institute of Technology; 2002.

26. Goodman RN, Rietschel JC, Roy A, Jung BC, Diaz J, Macko RF, Forrester LW. Oral presentation: The efficacy and mechanisms of robot-assisted therapies in neurorehabilitation. 10th Annual World Conference of the Society for Brain Mapping and Therapeutics; 2013 May; Baltimore, MD.
27. Bromberg-Martin ES, Matsumoto M, Hikosaka O. Dopamine in motivational control: rewarding, aversive, and alerting. Neuron. 2010;68(5):815-34. [PMID:21144997] http://dx.doi.org/10.1016/j.neuron.2010.11.022

28. Pleger B, Ruff CC, Blankenburg F, Klöppel S, Driver J, Dolan RJ. Influence of dopaminergically mediated reward on somatosensory decision-making. PLoS Biol. 2009;7(7): e1000164. [PMID:19636360] http://dx.doi.org/10.1371/journal.pbio.1000164

29. Hosp JA, Pekanovic A, Rioult-Pedotti MS, Luft AR. Dopaminergic projections from midbrain to primary motor cortex mediate motor skill learning. J Neurosci. 2011;31(7): 2481-87. [PMID:21325515] http://dx.doi.org/10.1523/JNEUROSCI.5411-10.2011

30. Kringelbach ML, Rolls ET. The functional neuroanatomy of the human orbitofrontal cortex: Evidence from neuroimaging and neuropsychology. Prog Neurobiol. 2004;72(5): 341-72. [PMID:15157726] http://dx.doi.org/10.1016/j.pneurobio.2004.03.006

31. LeDoux JE. The synaptic self: How our brains become who we are. New York (NY): Viking; 2002.

32. Schultheiss OC, Wirth MM, Waugh CE, Stanton SJ, Meier EA, Reuter-Lorenz P. Exploring the motivational brain: Effects of implicit power motivation on brain activation in response to facial expressions of emotion. Soc Cogn Affect Neurosci. 2008;3(4):333-43. [PMID:19015083] http://dx.doi.org/10.1093/scan/nsn030

33. Small DM, Gitelman D, Simmons K, Bloise SM, Parrish T, Mesulam MM. Monetary incentives enhance processing in brain regions mediating top-down control of attention. Cereb Cortex. 2005;15(12):1855-65. [PMID:15746002] http://dx.doi.org/10.1093/cercor/bhi063

34. Engelmann JB, Pessoa L. Motivation sharpens exogenous spatial attention. Emotion. 2007;7(3):668-74. [PMID:17683222] http://dx.doi.org/10.1037/1528-3542.7.3.668

35. Pessoa L. How do emotion and motivation direct executive control? Trends Cogn Sci. 2009;13(4):160-66. [PMID:19285913] http://dx.doi.org/10.1016/j.tics.2009.01.006

36. Scudder MR, Drollette ES, Pontifex MB, Hillman CH. Neuroelectric indices of goal maintenance following a single bout of physical activity. Biol Psychol. 2012;89(2): 528-31. [PMID:22200656]

http://dx.doi.org/10.1016/j.biopsycho.2011.12.009

37. Schmidt RA, Wrisberg CA. Motor learning and performance: A situation-based learning approach. Champaign (IL): Human Kinetics; 2008.

38. Vallerand RJ. Intrinsic and extrinsic motivation in sport and physical activity: A review and a look to the future. In: Tenebaum G, Eklund RC, editors. Handbook of sport psychology. 3rd ed. Hoboken (NJ): Wiley; 2007. 
39. Ghashghaei HT, Hilgetag CC, Barbas H. Sequence of information processing for emotions based on the anatomic dialogue between prefrontal cortex and amygdala. Neuroimage. 2007;34(3):905-23. [PMID:17126037] http://dx.doi.org/10.1016/j.neuroimage.2006.09.046

40. Tran AH, Tamura R, Uwano T, Kobayashi T, Katsuki M, Ono T. Dopamine D1 receptors involved in locomotor activity and accumbens neural responses to prediction of reward associated with place. Proc Natl Acad Sci USA. 2005;102(6):2117-22. [PMID:15684065] http://dx.doi.org/10.1073/pnas.0409726102

41. Siegert RJ, Taylor WJ. Theoretical aspects of goal-setting and motivation in rehabilitation. Disabil Rehabil. 2004; 26(1):1-8. [PMID:14660192] http://dx.doi.org/10.1080/09638280410001644932

42. Salomons TV, Johnstone T, Backonja MM, Davidson RJ. Perceived controllability modulates the neural response to pain. J Neurosci. 2004;24(32):7199-7203. [PMID:15306654] http://dx.doi.org/10.1523/JNEUROSCI.1315-04.2004

43. Lutz A, Greischar LL, Rawlings NB, Ricard M, Davidson RJ. Long-term meditators self-induce high-amplitude gamma synchrony during mental practice. Proc Natl Acad Sci USA. 2004;101(46):16369-73. [PMID:15534199] http://dx.doi.org/10.1073/pnas.0407401101

44. Lutz A, Lachaux JP, Martinerie J, Varela FJ. Guiding the study of brain dynamics by using first-person data: Synchrony patterns correlate with ongoing conscious states during a simple visual task. Proc Natl Acad Sci USA. 2002; 99(3):1586-91. [PMID:11805299]

http://dx.doi.org/10.1073/pnas.032658199

45. Hatfield BD, Haufler AJ, Hung TM, Spalding TW. Electroencephalographic studies of skilled psychomotor performance. J Clin Neurophysiol. 2004;21(3):144-56.

[PMID:15375345] http://dx.doi.org/10.1097/00004691-200405000-00003

46. Hatfield BD, Kerick SE. The psychology of superior sport performance. In: Tenebaum G, Eklund RC, editors. Handbook of sport psychology. 3rd ed. Hoboken (NJ): Wiley; 2007. p. 84-109.

47. Del Percio C, Infarinato F, Iacoboni M, Marzano N, Soricelli A, Aschieri P, Eusebi F, Babiloni C. Movement-related desynchronization of alpha rhythms is lower in athletes than non-athletes: A high-resolution EEG study. Clin Neurophysiol. 2010;121(4):482-91. [PMID:20097129] http://dx.doi.org/10.1016/j.clinph.2009.12.004

48. Perry J. Gait analysis: Normal and pathological function. Thorofare (NJ): SLACK; 1992.

49. Jasper HH. The ten-twenty electrode system of the international system federation. Electroencephalogr Clin Neurophysiol. 1958;10:371-75.
50. Onton J, Westerfield M, Townsend J, Makeig S. Imaging human EEG dynamics using independent component analysis. Neurosci Biobehav Rev. 2006;30(6):808-22.

[PMID:16904745]

http://dx.doi.org/10.1016/j.neubiorev.2006.06.007

51. Rohrer B, Fasoli S, Krebs HI, Volpe B, Frontera WR, Stein J, Hogan N. Submovements grow larger, fewer, and more blended during stroke recovery. Mot Control. 2004;8(4): 472-83. [PMID:15585902]

52. Coan JA, Allen JJ. Handbook of emotion elicitation and assessment. Oxford (England): Oxford University Press; 2007.

53. Rolls ET. The brain and emotion. Oxford (England): Oxford University Press; 1999.

54. Rolls ET. Emotion explained. Oxford (England): Oxford University Press; 2005.

55. Schultz W. Behavioral theories and the neurophysiology of reward. Annu Rev Psychol. 2006;57:87-115.

[PMID:16318590]

http://dx.doi.org/10.1146/annurev.psych.56.091103.070229

56. Fregni F, Pascual-Leone A. Hand motor recovery after stroke: Tuning the orchestra to improve hand motor function. Cogn Behav Neurol. 2006;19(1):21-33. [PMID:16633016] http://dx.doi.org/10.1097/00146965-200603000-00003

57. Serrien DJ, Strens LH, Cassidy MJ, Thompson AJ, Brown P. Functional significance of the ipsilateral hemisphere during movement of the affected hand after stroke. Exp Neurol. 2004;190(2):425-32. [PMID:15530881] http://dx.doi.org/10.1016/j.expneurol.2004.08.004

58. Fitts PM, Posner MI. Human performance. Belmont (CA): Brooks/Cole Publishing Co; 1967.

59. Mishra J, Zinni M, Bavelier D, Hillyard SA. Neural basis of superior performance of action videogame players in an attention-demanding task. J Neurosci. 2011;31(3):992-98. [PMID:21248123] http://dx.doi.org/10.1523/JNEUROSCI.4834-10.2011

60. Mirelman A, Bonato P, Deutsch JE. Effects of training with a robot-virtual reality system compared with a robot alone on the gait of individuals after stroke. Stroke. 2009;40(1): 169-74. [PMID:18988916] http://dx.doi.org/10.1161/STROKEAHA.108.516328

61. Onla-or S, Winstein CJ. Determining the optimal challenge point for motor skill learning in adults with moderately severe Parkinson's disease. Neurorehabil Neural Repair. 2008;22(4):385-95. [PMID:18326891] http://dx.doi.org/10.1177/1545968307313508

62. Guadagnoli MA, Lee TD. Challenge point: A framework for conceptualizing the effects of various practice conditions in motor learning. J Mot Behav. 2004;36(2):212-24. [PMID:15130871] http://dx.doi.org/10.3200/JMBR.36.2.212-224 
63. Mattia D, Spanedda F, Babiloni F, Romigi A, Marciani MG. Quantitative EEG patterns following unilateral stroke: A study in chronic stage. Int J Neurosci. 2003;113(4):465-82. [PMID:12856476] http://dx.doi.org/10.1080/00207450390162227

64. Nolfe G, Cobianchi A, Mossuto-Agatiello L, Giaquinto S. The role of P300 in the recovery of post-stroke global aphasia. Eur J Neurol. 2006;13(4):377-84. [PMID:16643316] http://dx.doi.org/10.1111/j.1468-1331.2006.01237.x

65. Mima T, Toma K, Koshy B, Hallett M. Coherence between cortical and muscular activities after subcortical stroke. Stroke. 2001;32(11):2597-2601. [PMID:11692023] http://dx.doi.org/10.1161/hs1101.098764

66. Strens LH, Asselman P, Pogosyan A, Loukas C, Thompson AJ, Brown P. Corticocortical coupling in chronic stroke: Its relevance to recovery. Neurology. 2004;63(3):475-84. [PMID:15304578] http://dx.doi.org/10.1212/01.WNL.0000133010.69694.F8

67. de Vico Fallani F, Astolfi L, Cincotti F, Mattia D, la Rocca D, Maksuti E, Salinari S, Babiloni F, Vegso B, Kozmann G,
Nagy Z. Evaluation of the brain network organization from EEG signals: a preliminary evidence in stroke patient. Anat Rec (Hoboken). 2009;292(12):2023-31.

[PMID:19943355]

http://dx.doi.org/10.1002/ar.20965

Submitted for publication February 26, 2013. Accepted in revised form September 23, 2013.

This article and any supplementary material should be cited as follows:

Goodman RN, Rietschel JC, Roy A, Jung BC, Diaz J, Macko RF, Forrester LW. Increased reward in ankle robotics training enhances motor control and cortical efficiency in stroke. J Rehabil Res Dev. 2014;51(2):213-28. http://dx.doi.org/10.1682/JRRD.2013.02.0050

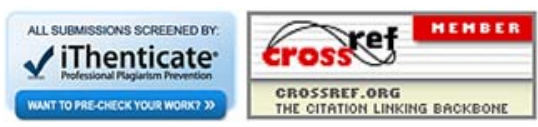


